\title{
A case report of retrograde suction decompression of a large paraclinoid aneurysm
}

\author{
Ahmed Mohamed Khalil ${ }^{1}$, Yashuhiro Yamada ${ }^{2}$, \\ Katsumi Takizawa ${ }^{3}$, Yoko Kato ${ }^{2}$, Ahmed Ansari ${ }^{2}$ \\ 1 Department of Neurosurgery, Faculty of Medicine, Alexandria \\ University, EGYPT \\ 2 Department of Neurosurgery, Bantane Hospital, Fujita Health \\ University, Nagoya, JAPAN \\ ${ }^{3}$ Department of Neurosurgery, Asahikawa Redcross Hospital, \\ Hokkaido, JAPAN
}

\begin{abstract}
Surgical clipping of large Paraclinoidal IC (Internal carotid) aneurysm pose a great challenge as there are adhesions hindering exposure of aneurysm dome and parent artery . Obtaining adequate visualization of the aneurysm neck is very difficult in these aneurysms, also in the access of proximal control. There are many methods to obtain a proximal control in these aneurysms. Retrograde suction decompression provides adequate visualization of the aneurysm neck and its relation with the optic apparatus. Retrograde suction decompression can be done by many methods. The technique done via open catheterization of superior thyroid artery is readily accessible and provides adequate relaxation of the aneurysm dome enabling complete dissection of the aneurysm from the surrounding important neurovascular structures. In this report of a case of left Paraclinoidal ICA aneurysm which was clipped applying this method, we elaborate on the technique and discuss other methods available for proximal control in these difficult aneurysms.
\end{abstract}

\section{INTRODUCTION}

Microsurgical treatment for paraclinoid aneurysms continue to pose severe challenges to vascular neurosurgeons because of attaining difficulty in proximal control of the parent artery and obtaining adequate visualization of the aneurysm neck. (1)

Microsurgical treatment of large paraclinoid aneurysms often requires the use of the one or the other methods of attaining proximal control. These can vary from exposure of internal carotid artery in the neck, Adenosine induced cardiac arrest, Rapid ventricular pacing (RVP), and Retrograde suction decompression (RSD). These, in addition to anterior clinoidectomy facilitate safe and complete clipping in these difficult aneurysms. $(2,3)$

RSD can be in the form of both open as well as endovascular approach. In both variants it provides adequate relaxation of the
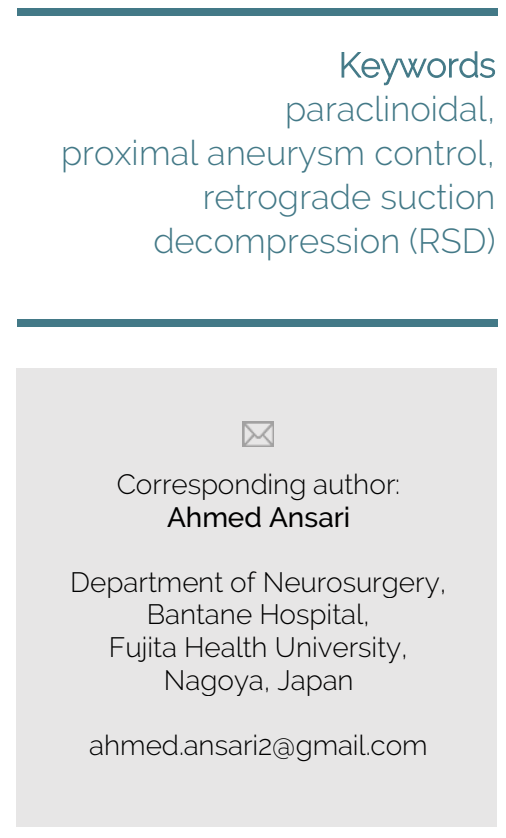

Copyright and usage. This is an Open Access article, distributed under the terms of the Creative commons Attribution Non-Commercial No Derivatives License (https://creativecommons org/licenses/by-nc-nd/4.0/) which permits noncommercial re-use, distribution, and reproduction in any medium, provided the original work is unaltered and is properly cited.

The written permission of the Romanian Society of Neurosurgery must be obtained for commercial re-use or in order to create a derivative work.

ISSN online 2344-4959

(C) Romanian Society of Neurosurgery

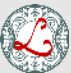

First published

September 2019 by

London Academic Publishing wwww.lapub.co.uk 
aneurysm dome, enabling the surgeon to dissect the aneurysmal complex from the surrounding structures such as optic apparatus and dural ring allowing adequate visualization of the aneurysm neck and reconstruction of the parent artery. $(1,4,5$, 6)

We present a case and discuss the surgical nuances of retrograde suction decompression of a large left Paraclinoidal IC aneurysm in Banbuntane Hotokukai Hospital, Fujita Health University, Japan. Haemorrhages or eponymously Duret haemorrhages are a feared consequence of a transtentorial brain herniation or of a decompression of the intracranial space by means of craniectomy or by removal of an intracranial mass lesions. $(16,21,22)$.

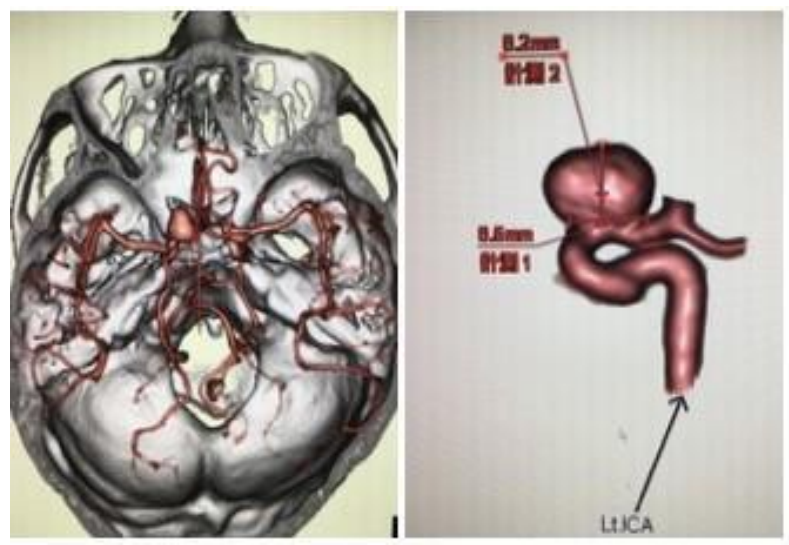

FIGURE 1. 3D-CTA Show left Paraclinoidal aneurysm with fundus height $8.2 \mathrm{~mm}$, neck width $8.5 \mathrm{~mm}$

\section{OPERATIVE PROCEDURE}

The patient was placed in supine position with head rotated 30 degrees and neck slightly extended to facilitate cervical incision. Intraoperative monitoring MEP was used. A curvilinear incision of about $10 \mathrm{~cm}$ was made in the neck. Deeper layers were exposed in succession to dissect out the common carotid, external and internal carotid artery. The superior thyroid artery was dissected and secured with a loop. A standard left pterional craniotomy in addition to anterior clinoidectomy (extradural) was done. The sylvian fissure was widely split for minimal retraction of the frontal lobe to expose the internal carotid artery and the optic nerve. The large aneurysm arising from the medial wall of the carotid was visualized. The posterior communicating artery, anterior choroid artery (Ach), and their branches were also defined. The common carotid, external

\section{CASE REPORT}

A 49-year-old woman was admitted to our hospital with incidentally detected, no history of headache, dizziness or blurring of vision. Magnetic resonance angiography showed a left Paraclinoidal aneurysm. Three-dimensional computed tomography (CT) angiography (3D-CTA) and digital subtraction angiography revealed a left Paraclinoidal aneurysm with a fundus height $8.2 \mathrm{~mm}$ in size, neck width $8.5 \mathrm{~mm}$ (Figure 1). We also performed computational fluid dynamics (CFD) which showed wall pressure to be high, wall shear stress was low and vectors were divergent at the base of neck as it impend rupture (Figure 2).

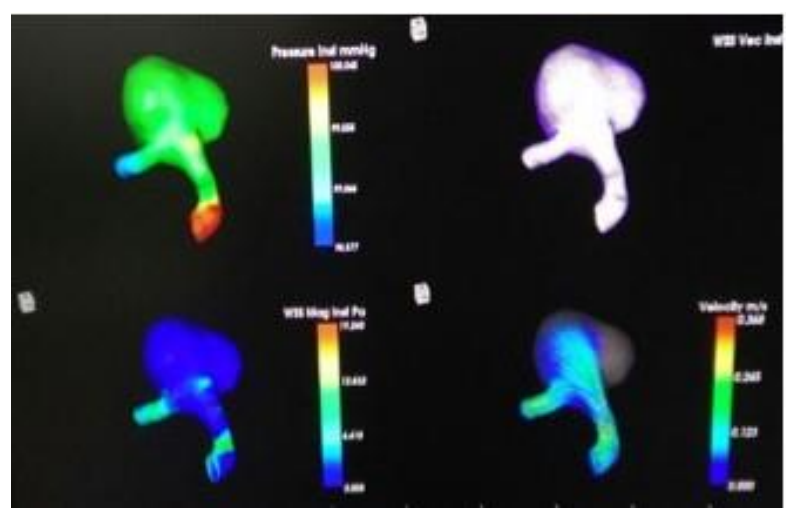

FIGURE 2: CFD of our left Paraclinoidal aneurysmthese

carotid distal to superior thyroid artery and internal carotid artery distal to the aneurysm were clamped. The superior thyroid artery was opened and a $5 \mathrm{~F}$ feeding tube was introduced in the direction of the internal carotid artery. Retrograde suction decompression (RSD) was performed with the help of 10cc syringe. The aneurysm was effectively deflated following which a definitive clipping of aneurysm was performed with two permanent clips (Figure 3). Post clipping dual injection video angiography (DIVA) showed complete occlusion of the aneurysm (Figure 4). The postoperative was uneventful and patient recovered well without any deficits. 

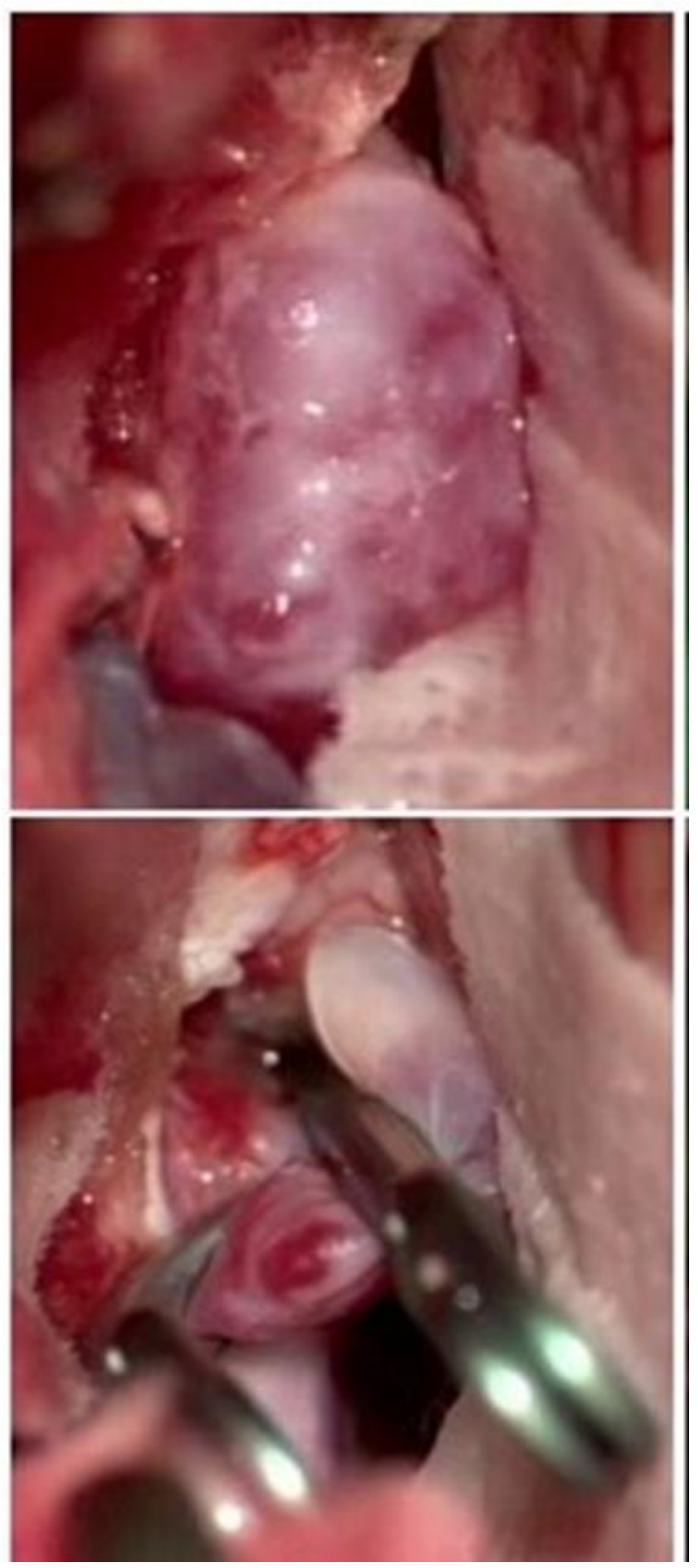

FIGURE 3: show defensive clipping of left Paraclinoidal aneurysm with 2 permanent clips

\section{DISCUSSION}

Paraclinoidal aneurysms are surrounded by many important osseous and neurovascular structures, which continue to present great difficulties in achieving proximal control of the parent artery and obtaining adequate visualization of the aneurysm neck because of adhesion to surrounding anatomical components $(7,8)$. Therefore, anterior clinoidectomy and dissection of the neurovascular components from the aneurysm dome are the key steps to safe and successful clipping surgery for these aneurysms. In this scenario, the importance of proximal control of the aneurysm becomes
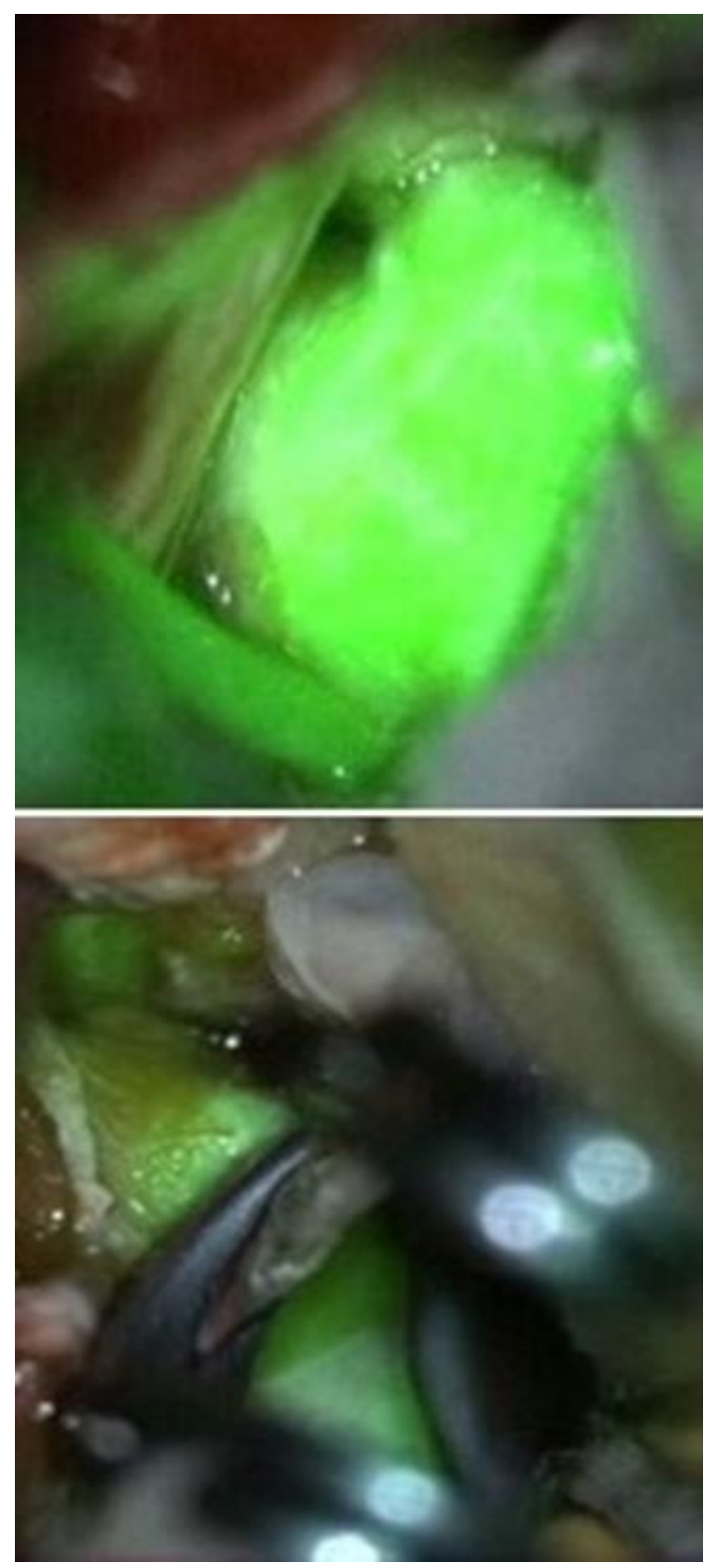

FIGURE 4. DIVA showed complete occlusion of aneurysm

indispensable. Among the several options for this RSD technique provides adequate relaxation of the aneurysm dome enabling complete dissection of the aneurysm from the surrounding important neurovascular structures $(1,4$, and 6$)$

Retrograde suction aspiration was first described by Bather and Samson, who inserted an angiocatheter into the cervical ICA and treated over 40 cases with giant paraclinoid aneurysms using this technique. Carotid artery dissection developed in a case in their series, which required emergency endarterectomy. (7) Tamaki, et al reported the use of the RSD technique under the name of 'trapping- 
evacuation.' They deflated aneurysms by aspirating intra-aneurysmal blood through the ICA via the superior thyroid artery (8).

In our case, we use RSD via catheterization of superior thyroid artery with clamping common carotid artery, external carotid distal to it and internal carotid artery distal to Paraclinoidal aneurysm. So, it allowed dissection, definitive clipping and reconstruction of internal carotid artery.

This measure can be done using endovascular balloon but it had a risk of embolization and dissection of internal carotid artery. It has its advantages in that, it avoid neck incision and also allows performing intra-operative angiography $(9$, 10).

Other measures described for the proximal control of aneurysms are adenosine assisted cardiac arrest, Rapid ventricular pacing. Adenosine induced arrest allows temporary flow arrest into the parent vessel, facilitating the circumferential exposure of the aneurysm and decreasing the risk of premature rupture during the dissection, and finally safe clip placement but it had drawbacks like causing atrial fibrillation ,bronchospasm and can be perilous in medically ill patient(11,12).Also, Rapid ventricular placing (RVP) was to induce hypotension in the face of sudden intraoperative hemorrhage which could not be controlled with conventional means during intracranial aneurysm surgery. Unlike adenosine, the consideration to use RVP is not affected by patient factors including allergies or medical conditions such as asthma and heart blocks (13).

\section{CONCLUSION}

The RSD technique viacatheterization of superior thyroid artery provides adequate relaxation of the aneurysm dome enabling complete dissection of the aneurysm from the surrounding important neurovascular structures and also avoids dissection of internal carotid artery and frequent embolization, so it is an important surgical tool in the surgery for large paraclinoid aneurysms.

\section{REFERENCES}

1. Fulkerson DH, Horner TG, Payne TD, Leipzig TJ, Scott JA, Leonardo AJ, et al. Results, outcomes, and follow-up of remnants in the treatment of ophthalmic aneurysms: A 16year experience of a combined neurosurgical and endovascular team. Neurosurgery. 2009; 64:218-29.

2. DayJD, Gannett SL, Fukushima T. Extradural temporopolar approach to lesions of the upper basilar artery and infrachiasmatic region. J Neurosurg. 1994; 81:230-5.

3. Day JD, Fukushima T, Gannett SL. Cranial base approaches to posterior circulation aneurysms. J Neurosurg. 1997; 87:544-54.

4. Bather HH, Samson DS. Retrograde suction decompression of giant Paraclinoidal aneurysms. Technical note. J Neurosurg. 1990; 73:305-6.

5. Fan YW, Chan $\mathrm{KH}$, Luis WM, and Hung KN. Retrograde suction decompression of paraclinoid aneurysm - A revised technique. Surge Neurol. 1999; 51:129-31.

6. Miso K, Takahashi A, Yoshimoto T, Fujiwara S, Kosher K. Combined endovascular and neurosurgical approach for paraclinoid internal carotid artery aneurysms. Neurosurgery. 1993; 33:986-92.

7. Bather HH, Samson DS. Retrograde suction decompression of giant Paraclinoidal aneurysms. Technical note. J Neurosurg 1990; 73:305-6.

8. Tamaki N, Kim S, Hear K, Asada M, Fujita K, Tomato K, et al. Giant carotid-ophthalmic artery aneurysms: direct clipping utilizing the "trapping-evacuation" technique. J Neurosurg 1991;74:567-72

9. Hoh DJ, Larsen DW, Elder JB, Kim PE, Gannett SL, Liu CY. Novel use of an endovascular embolectomy device for retrograde suction decompression-assisted clip ligation of a large paraclinoid aneurysm: technical case report. Neurosurgery 2008;62(5 Suppl 2): ONSE412-3.

10. Fulkerson DH, Horner TG, Payne TD, Leipzig TJ, Scott JA, Edgardo $\mathrm{A}$, et al. Endovascular retrograde suction decompression as an adjunct to surgical treatment of ophthalmic aneurysms: analysis of risks and clinical outcomes. Neurosurgery 2009; 64(3 Suppl):ons107-11.

11. Beady JF, Gupta DK, Benda BR, Hemmer LB, Zen C, Aram $M J$, et al. Adenosine-induced flow arrest to facilitate intracranial aneurysm clip ligation: Dose-response data and safety profile. Anesth Analg 2010;110:1406-11.

12. Guinn NR, McDonagh DL, Borel CO, Wright DR, Zomorodi AR, Powers CJ, et al. Adenosine-induced transient asystole for intracranial aneurysm surgery: A retrospective review. J Neurosurg Anesthesiol 2011;23:35-40.

13. Davidavicius G, Chieffo A, Shannon J, Arioli F, lelasi A, Mussardo $M$, et al. $A$ high dose of adenosine to induce transient asystole for valvuloplasty in patients undergoing transcatheter aortic valve implantation (TAVI): Is it a valid alternative to rapid pacing? A prospective pilot study. J Invasive Cardiol. 2011;23:467-71. 